\title{
Mathematical modeling of drive transitive process with linear change in control input
}

\author{
Dmitriy Ershov ${ }^{1,2}$, Irina Lukyanenko ${ }^{1}$ and Evgeniy Zlotnikov ${ }^{2}$ \\ ${ }^{1}$ Saint-Petersburg University of Aerospace Instrumentation (SUAI), 67, BolshayaMorskaia str., St Petersburg, \\ 190000, Russia \\ ${ }^{2}$ Saint-Petersburg Mining University, 2, 21st Line, St Petersburg 199106, Russia
}

\begin{abstract}
The paper researches into dynamic parameters of transitive processes in electrical drive of technological equipment in acceleration mode given changes in control input under law of linearity. Analysis of two-mass dynamic model allows for solutions of differential equations describing three stages in the drive acceleration mode. Dynamic and static components of drive errors are discussed in relation to its velocity as being conditioned on the moment of load, the total moment of the drive inertia, stiffness ratio of static parameter and pre-defined programmed acceleration.
\end{abstract}

\section{Keywords}

Technological equipment, transitive process, drive acceleration, motor angular velocity

\section{Introduction}

Conditions under which manufacturing and industrial equipment operates require setting a process of transition either to produce a pre-defined principle of changes in actuator angular velocity, or as a requirement for pre-determined speed of equipment operation, or to produce limited and controlled dynamic loads. The required parameters determining transitive process can be achieved by setting the law for changes in control input-voltage $U_{c}(t)$, which is transferred to the armature. This law is made operational by a specialized transducer.

\section{Mathematical Modeling}

The control input that is changed linearly can be described by the following equation:

$$
\frac{U_{c}}{k F}(t)=\omega_{0}(t)=\varepsilon_{p} t
$$

where $F$ is magnetic flux, $k$ is designed parameter of motor, $\varepsilon_{p}$ is the pre-determined value of programmed acceleration provided by transducer operation.

The moment of motor can be given as:

$$
M_{m}=\beta_{m} \varepsilon_{p} t-\beta_{m} \omega_{m}
$$

The dynamic model is given in Figure 1:.

Differential equations for drive motion are done as:

$$
\begin{gathered}
J_{m} \dot{\omega}_{m}=M_{m}+M_{12}, \\
J_{i} \dot{\omega}_{i}=M_{21}-M_{i}^{0}, \\
M_{m}=\beta_{m} \varepsilon_{p} t-\beta_{m} \omega_{m}
\end{gathered}
$$

III International Workshop on Modeling, Information Processing and Computing (MIP: Computing-2021), May 28, 2021, Krasnoyarsk, Russia

EMAIL: fetcat@mail.ru (Dmitriy Ershov); irina.n.lukyanenko@gmail.com (Irina Lukyanenko); zlotnik_evg@mail.ru (Evgeniy Zlotnikov) ORCID: 0000-0001-9384-7590 (Dmitriy Ershov); 0000-0002-9647-0781 (Evgeniy Zlotnikov)

\footnotetext{
(c) (i) (C) 2021 Copyright for this paper by its authors.

Use permitted under Creative Commons License Attribution 4.0 International (CC BY 4.0).

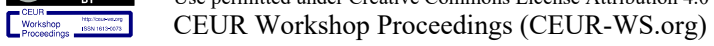


Analysis and description of the drive in acceleration mode requires determining the static mechanical parameter of the motor $[1,2,7]$, with the moment of load considered a constant. The above system can be reduced to one equation in relation to $\omega_{m}$.

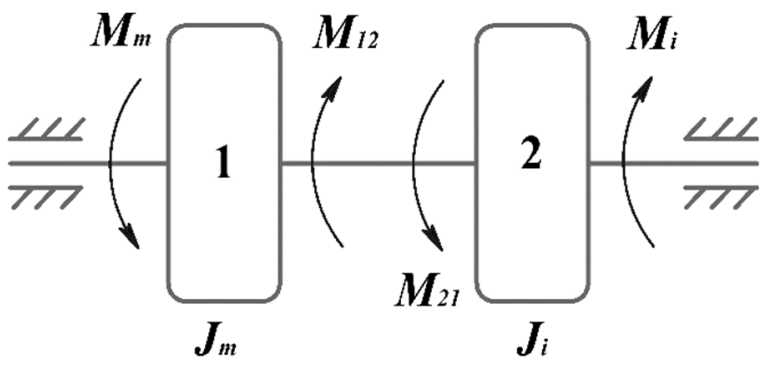

Figure 1: Dynamic model of technological drive

$$
\left(J_{m}+J_{i}\right) \dot{\omega}_{m}+\beta_{m} \omega_{m}=\beta_{m} \varepsilon_{p} t-M_{i}^{0}
$$

At the initial point in acceleration given $t=0 \omega_{m}=0$, the control input given (1) equals zero $\omega_{0}(0)=0$ and consequently given (2) the moment of motor also equals zero. Hence, (3) conditions the drive motion to be possible only if the moment of motor $M_{m}$ has values higher than the moment of load $M_{i}^{0}$ . The mode of acceleration is characterized by a stage when the moment of motor is continuously increasing with time while mechanical system remains static. This stage can be described by the following equation:

$$
\beta_{m} \varepsilon_{p} t-M_{i}^{0}=0
$$

This first stage finishes with $t=t_{1}$, for which the condition of $M_{m}\left(t_{1}\right)=\beta_{m} \varepsilon_{p} t_{1}=M_{i}^{0}$ holds. It produces $t_{1}=\frac{M_{i}^{0}}{\beta_{m} \varepsilon_{p}}$.

At the second stage in acceleration mode, given $t>t_{1}$, the mechanical system is set in motion and the moment of motor is described as:

$$
M_{m}(t)=\beta_{m} \varepsilon_{p}\left(t-t_{1}\right)+M_{i}^{0}-\beta_{m} \omega_{m} .
$$

The equation describing the system motion in the second stage can be done as:

where $T_{m}=\left(J_{m}+J_{i}\right) \beta_{m}^{-1}$.

$$
T_{m} \dot{\omega}_{m}+\omega_{m}=\varepsilon_{p}\left(t-t_{1}\right),
$$

Solution to equation (3) produces equation which determines changes in angular velocity at the second stage of acceleration mode:

$$
\omega_{m}(t)=\varepsilon_{m}\left(t-t_{1}\right)-T_{m} \varepsilon_{p}\left(1-e^{-\frac{t-t_{1}}{T_{m}}}\right) .
$$

Given dependence (1), relation of $\varepsilon_{p} t_{1}=\frac{M_{i}^{0}}{\beta_{m}}$, produces:

$$
\omega_{m}(t)=\omega_{0}(t)-\frac{M_{i}^{0}}{\beta_{m}}-\frac{\left(J_{m}+J_{i}\right) \varepsilon_{p}\left[1-e^{-\frac{t-t_{1}}{T_{m}}}\right]}{\beta_{m}} .
$$

The value of $\Delta \omega_{m}^{0}=\frac{M_{i}^{0}}{\beta_{m}}$ determines reductions in the angular velocity value in relation to the preset value $\omega_{0}(t)$, determined at the drive input and conditioned on the moment of load $M_{i}^{0}$ - this produces drive velocity static error. The value of $M_{m}^{d}=\left(J_{m}+J_{i}\right) \varepsilon_{p}$ determines the dynamic component of moment $M_{m}$ generated by the motor and providing conditions for the drive acceleration with the pre- 
determined acceleration rate $\varepsilon_{p}$. The third addend in (5) is given as reduction in the angular velocity in relation to pre-determined value $\omega_{0}(t)$, conditioned on the dynamic load and considered drive velocity dynamic error. The value of $\Delta \omega_{m}^{d}=\frac{\left(J_{m}+J_{i}\right) \varepsilon_{p}}{\beta_{m}}=T_{m} \varepsilon_{p}$ is regarded the pre-determined value of dynamic error. The resulting function (5) can be given as:

$$
\omega_{m}(t)=\omega_{0}(t)-\Delta \omega_{m}^{0}-\Delta \omega_{m}^{d}\left(1-e^{-\frac{t-t_{1}}{T_{m}}}\right) .
$$

Given $t=t_{2}=3 T_{m}+t_{1}$ the function $e^{-\frac{t-t_{1}}{T_{m}}}=0,05$ and then given $t>t_{2}$ the angular velocity $\omega_{m}(\mathrm{t})$ can be described as:

$$
\omega_{m}(t)=\omega_{0}(t)-\Delta \omega_{m}^{0}-\Delta \omega_{m}^{d}
$$

The second stage of the drive acceleration mode is completed in time $\mathrm{t}=\mathrm{t} 3$, in which the angular velocity in idle running $\omega_{0}$ reaches a certain pre-determined value $\omega_{0}^{*}$. Time moment $t_{3}$ is determined in the following manner: $t_{3}=\frac{\omega_{0}^{*}}{\varepsilon_{p}}$.

The third stage of the drive acceleration mode, when $t>t_{3}$, can be described with differential equation (3) done as:

$$
\left(J_{m}+J_{i}\right) \dot{\omega}_{m}+\beta_{m} \omega_{m}=\beta_{m} \omega_{0}^{*}-M_{i}^{0}
$$

Dividing all components of equation by $\beta_{m}$ produces the following equation:

$$
T_{m} \dot{\omega}_{m}+\omega_{m}=\omega_{0}^{*}-M_{i}^{0} \beta_{m}^{-1}
$$

The difference $\omega_{0}^{*}-M_{i}^{0} \beta_{m}^{-1}$ determines the values of angular velocity $\omega_{m}^{0}$ under the steady-state mode of drive motion. Differential equation describing the third stage of the drive acceleration mode can be done as:

$$
T_{m} \dot{\omega}_{m}+\omega_{m}=\omega_{m}^{0}
$$

Solution to the last equation produces condition according to which the value of angular velocity at this stage is determined by the function:

$$
\omega_{m}(t)=\omega_{m}^{0}-\Delta \omega_{m}^{d} e^{-\frac{t-t_{3}}{T_{m}}}=\omega_{m}^{0}-T_{m} \varepsilon_{p} e^{-\frac{t-t_{3}}{T_{m}}}
$$

Figure 2: demonstrates the diagrams for dependencies $\omega_{0}(t)=\varepsilon_{p} t, \omega_{0}(t)=\varepsilon_{p}\left(t-t_{1}\right)$, $\omega_{0}(t)=\omega_{m}(t)$. The curve $a b c$ is plotted based on (4). When the point of the curve in which $t=t_{1}$ reached, the drive mechanical system is set in motion, transiting from static to dynamic mode. Given this condition, velocity time error $t$ equals $\omega_{m}^{0}$. It is determined by the value of the moment of load $M_{i}^{0}$ and stiffness ratio of static parameter $\beta_{m}$. Given changes in time value $t$ in the range of $t_{1}<t<t_{2}$ acceleration of the drive mechanical system is determined by region $a b$ of the curve in which the total error in velocity is increased by the dynamic components rising in the range of 0 to the steady-state value of $\Delta \omega_{m}^{d}$. Within this time range the angular velocity value is determined by dependencies (4) - (6). Given $t>t_{2}$ the drive acceleration mode is determined by region $b c$ of the curve, in which the value of angular velocity is described by function (7). The steady-state value of dynamic error in velocity $\Delta \omega_{m}^{d}$ is the higher the higher the total moment of the drive inertia $J_{m}+J_{i}$ and the pre-determined programmed acceleration $\varepsilon_{p}$, and is the lower the higher $\beta_{m}$.

The diagram of dependence of equation (8) is shown with the curve $c d$. The value of angular velocity $\omega_{m}(t)$ asymptotically approaches the value of $\omega_{m}^{0}$, which determines the drive angular velocity in steady-state mode of drive motion. Given $t>t_{4}=3 T_{m}+t_{3}$ angular velocity $\omega_{m}(t)$ is different from the value $\omega_{m}^{0}$ by less than $5 \%$, and transitive process is considered completed. Under the steady-state mode of the drive operation drive velocity static error $\Delta \omega_{m}^{0}$ preserves. 


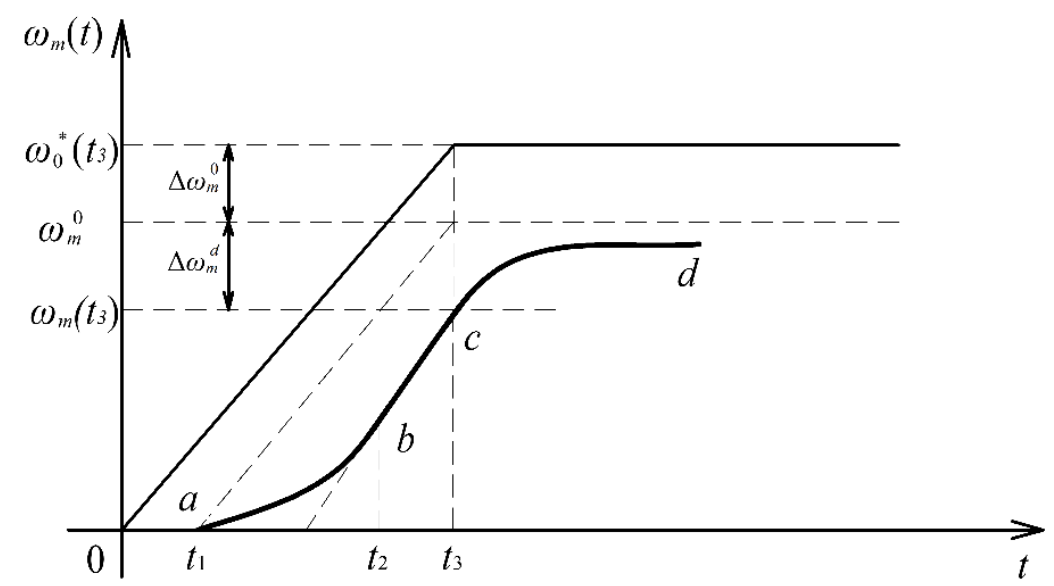

Figure 2: Diagram of the motor angular velocity dependence

The $t_{4}$ time range of transitive process of the third stage in drive acceleration is then calculated as:

$$
t_{4}=3 T_{m}+t_{3}=3 T_{m}+\frac{\omega_{0}^{*}}{\varepsilon_{p}}
$$

\section{Conclusion}

It follows from expression (9) that reductions in time intervals required for transitive process or increases in performance speeds of the drive can be gained by lower values of mechanical constant $T_{m}$ and higher values of programmed acceleration $\varepsilon_{p}$. Observance of the first condition requires either reduced values of total moment of inertia or increased values of stiffness ratio of static parameter $\beta_{m}$. Observance of the second condition requires increases in values of the motor starting torque which is subjected to a number of limitations conditioned on the parameters of the motor operation [3-6, 8-15].

\section{References}

[1] D. Y. Ershov, E. G. Zlotnikov, D. D. Timofeev, Analysis of proper fluctuations of technological systems, IOP Conference Series: Materials Science and Engineering 560(1) (2019) 12015-12015.

[2] D. Y. Ershov, E. G. Zlotnikov, B. Nestorovski, Own fluctuations of technological systems, Journal of Physics: Conference Series 1399 (2019) 22055-22055.

[3] V. V. Maksarov, A. D. Khalimonenko, J. J. Olt, Managing the process of machining on machines on the basis of dynamic modelling for a technological system, Epitoanyag-journal of silicate based and composite materials 2(69) (2017) 66-71.

[4] V. V. Maksarov, R.V. Vyushin, A. E. Efimov, Technological provisions for rough surface based on modeling transitive processes, Metalworking 2 (2017) 39-46.

[5] V. V. Maksarov, A. E. Efimov, D. Y. Timofeev, Modeling dynamic processes at stage of formation of parts previously subjected to high-energy laser effects, IOP Conference Series: Materials Science and Engineering 2(327) (2018) 22-25.

[6] J. J. Olt, V.V. Maksarov, A. E. Efimov, Improving quality of critical tractor parts through dynamic stabilization of manufacturing process on CNC machines, Agronomy Research 17(1) (2019)11461154.

[7] D. Y. Ershov, I. N. Lukjanenko, Mathematical modelling for analysis of self-locking mechanical systems operation in dynamic modes, Journal of Physics. Conf. Series, 1399(2) (2019) 2204322043.

[8] V. V. Maksarov, J. Olt, Improving the precision of manufacturing power hydraulic cylinders of powered roof supports based on a vibration-damping tooling system, Journal of Mining Institute 214 (2015) 71-84.

[9] V. V. Maksarov, J. Olt, Dynamic stabilization of machining process based on local metastability in controlled robotic systems of CNC machines, Journal of Mining Institute 226 (2017)446-451. 
[10] L. B. Alekseeva, Use of mechanical models for the analysis of antivibration mounting, IOP Conf. Series: Earth and Environmental Science 194(2) (2018) 22001.

[11] L. B. Alekseeva, Determination of transient behavior characteristics in drive control of machines, Mining Informational and Analytical Bulletin 4 (2016) 18-25.

[12] O. M. Bolshunova, A. A. Korzhev, A. M. Kamyshyan, Adaptive control system of dump truck traction electric drive, IOP Conference Series: Materials Science and Engineering, 327(5) (2018) 052007.

[13] A. A. Korzhev, O. M. Bolshunova, I. N. Voytyuk, A. Vatlina, Mathematical simulation of transient operation modes of an electric drive of a centrifugal pump for a slurry pipeline, E3S Web of Conferences 140 (2019). DOI: 10.1051/e3sconf/201914004012.

[14] E. K. Eshchin, Calculations of dynamic operating modes of electric drives of self-propelled mining machines, Journal of Mining Institute 233 (2018) 534-538.

[15] D. I. Shishlyannikov, A. A. Rybin, Assessment of load of beam-balanced pumping units by electric motor power indicators, Journal of Mining Institute 227 (2017) 582. 